\title{
The least-squares ambiguity decorrelation adjustment: its performance on short GPS baselines and short observation spans
}

\author{
P. J. G. Teunissen, P. J. de Jonge, C. C. J. M. Tiberius \\ Delft Geodetic Computing Centre (LGR), Faculty of Geodetic Engineering, Delft University of Technology, Thijsseweg 11, 2629 JA Delft, \\ The Netherlands Fax: +31 15 2783711; e-mail: lgr@geo.tudelft.nl
}

Received: 31 October 1995 / Accepted: 21 March 1997

\begin{abstract}
The least-squares ambiguity decorrelation adjustment is a method for fast GPS double-difference (DD) integer ambiguity estimation. The performance of the method will be discussed, and although it is stressed that the method is generally applicable, attention is restricted to short-baseline applications in the present contribution. With reference to the size and shape of the ambiguity search space, the volume of the search space will be introduced as a measure for the number of candidate grid points, and the signature of the spectrum of conditional variances will be used to identify the difficulty one has in computing the integer DD ambiguities. It is shown that the search for the integer leastsquares ambiguities performs poorly when it takes place in the space of original DD ambiguities. This poor performance is explained by means of the discontinuity in the spectrum of conditional variances. It is shown that through a decorrelation of the ambiguities, transformed ambiguities are obtained which generally have a flat and lower spectrum, thereby enabling a fast and efficient search. It is also shown how the high precision and low correlation of the transformed ambiguities can be used to scale the search space so as to avoid an abundance of unnecessary candidate grid points. Numerical results are presented on the spectra of conditional variances and on the statistics of both the original and transformed ambiguities. Apart from presenting numerical results which can typically be achieved, the contribution also emphasizes and explains the impact on the method's performance of different measurement scenarios, such as satellite redundancy, single vs dualfrequency data, the inclusion of code data and the length of the observation time span.
\end{abstract}

Key words. GPS $\cdot$ Ambiguity resolution $\cdot$ Ambiguity decorrelation

Correspondence to: P. J. G. Teunissen

\section{Introduction}

The GPS double-difference (DD) carrier-phase measurements are ambiguous by an unknown integer number of cycles. The a priori knowledge of the integer nature of the ambiguities can be used to strengthen the baseline solution. This is of particular relevance to applications where use is made of short observation time spans. No deterministic method exists for the computation of the most likely integer ambiguity estimates. In the literature, many important contributions have already been made in the area of GPS integer ambiguity estimation. Examples of proposed methods, together with suggested refinements, can be found in e.g. Blewitt (1989), Dong and Bock (1989), Frei and Beutler (1990), Hatch (1991), Frei (1991), Wübbena (1991) and Euler and Landau (1992).

An alternative method for fast GPS integer ambiguity estimation was introduced in Teunissen (1993). It makes use of a sequential conditional least-squares search, based on transformed ambiguities. The principles of the least-squares ambiguity decorrelation adjustment (LAMBDA) were discusssed in detail in Teunissen (1995a). An elementary presentation of the basic principles of the method is given in Teunissen et al. (1995), and a statistically based validation of the GPS baselines obtained with the method is given in Tiberius and De Jonge (1995). In this contribution, it is the performance of the method that will be discussed. The numerical results presented are results that can typically be achieved with the method. The results will be substantiated by means of a qualitative analysis of the method's performance.

The discussion of the method's performance will be restricted to applications where use is made of short baselines. For instance, the ionospheric delays have been assumed absent or sufficiently small to be neglected. The model used for our analysis is therefore the 'standard' model for short-baseline applications. In it, only the baseline components and integer DD ambiguities appear 
as unknown parameters. It should be noted, however, that this restriction does not stem from the method itself. If needed and when estimable, additional parameters (e.g. different types of delay, such as atmospheric and/or instrumental delays) can be included without affecting the principle of the method. The method is also not restricted to the use of single baselines only. Multiple baselines, which are simultaneously adjusted, can be handled too.

In order to make this contribution sufficiently selfcontained, we give a brief review of the steps involved in integer ambiguity estimation. As a starting point, we take the linearized system of observation equations

$y=A a+B b+e$

where:

$y \quad$ is the vector of observed minus computed DD carrier-phase measurements, possibly including code measurements as well;

$a$ is the vector of unknown integer DD ambiguities;

$b \quad$ is the vector that contains the increments of the unknown baseline components;

$A, B$ are the design matrices for ambiguity terms and baseline components, respectively;

$e \quad$ is the vector of measurement noise and unmodelled errors.

Note that we have followed the customary practice of working with the DD version of the carrier-phase observation equations. This is not really necessary, however, as is explained in Blewitt (1989), Teunissen (1995a) and Goad (1985). In fact, in our software we work with the undifferenced carrier phases and use a reparametrization on the original non-integer ambiguities so as to obtain integer ambiguities.

Since our estimation criterion will be based on the principle of least-squares, estimates for the unknown parameters of Eq. (1) follow from solving the minimization problem

$\min _{a, b}\|y-A a-B b\|_{Q_{y}}^{2}$ with $a \in Z^{n}, b \in R^{3}$

where $\|.\|_{O_{v}}^{2}=(.)^{*} Q_{y}^{-1}($.$) and Q_{y}$ is the variance-covariance matrix of the observables (the asterisk denotes the transpose). This minimization problem was referred to as an integer least-squares problem in Teunissen (1993). It is a constrained least-squares problem, due to the integer constraint $a \in Z^{n}$. The solution of the integer least-squares problem will be denoted as $\check{a}$ and $\breve{b}$. The solution of the corresponding unconstrained leastsquares problem will be denoted as $\hat{a}$ and $\hat{b}$. The estimates $\hat{a}$ and $\hat{b}$ are sometimes also referred to as the 'float solution', and the estimates $\check{a}$ and $\check{b}$ as the 'fixed solution'.

As it was shown in Teunissen (1993), the objective function of Eq. (2) can be decomposed into the following sum of $(n+2)$ squares

$\|y-A a-B b\|_{Q_{y}}^{2}=e_{1}+e_{2}+e_{3}$

with

$$
\begin{aligned}
& e_{1}=\|\hat{e}\|_{Q_{y}}^{2} \\
& e_{2}=\sum_{i=1}^{n}\left(\hat{a}_{i \mid I}-a_{i}\right)^{2} / \sigma_{\hat{a}_{i \mid I}}^{2} \\
& e_{3}=\|\hat{b} \mid a-b\|_{Q_{b \mid a}}^{2}
\end{aligned}
$$

and where

$\hat{e}=y-A \hat{a}-B \hat{b}$

$\hat{a}_{i \mid I}=\hat{a}_{i}-\sum_{j=1}^{i-1} \sigma_{\hat{a}_{i, j J}} \sigma_{\hat{a}_{\mid J}}^{-2}\left(\hat{a}_{j \mid J}-a_{j}\right)$

$\hat{b} \mid a=\hat{b}-Q_{\hat{b} \hat{a}} Q_{\hat{a}}^{-1}(\hat{a}-a)$

In this decomposition, $\hat{e}$ is the unconstrained leastsquares residual vector, $\hat{a}_{i||}$, with $I=\{1, \ldots,(i-1)\}$, is the least-squares estimate of the $i$-th ambiguity conditioned on $a_{1}$ up to and including $a_{i-1}$, and $\hat{b} \mid a$ is the least-squares estimate of the baseline vector conditioned on $a$. The variance of $\hat{a}_{i \mid I}$ is denoted as $\sigma_{\hat{a}_{||}}^{2}$, and the covariance between $\hat{a}_{i}$ and $\hat{a}_{j \mid J}$ is denoted as $\sigma_{\hat{a}_{i, j}}$. The estimates $\hat{a}_{i \mid l}, i=1, \ldots, n$ follow from a sequential conditional least-squares adjustment on the ambiguities. As shown in Teunissen (1993, 1995a), this corresponds algebraically to using a triangular decomposition of the variance-covariance matrix of the ambiguities.

In those GPS applications which are based on short observation time spans, the bottleneck in finding the solution of Eq. (2) is given by the problem of efficiently finding the minimizers of $e_{2}$ in Eq. (3), for $a_{i} \in Z$. The approach taken with the LAMBDA method, is to reparametrize the integer least-squares problem such that an equivalent problem is obtained, but one that is much easier to solve. It consists of two steps. First, an ambiguity transformation $Z^{*}$ is constructed that tries to decorrelate the ambiguities. In the construction of $Z^{*}$, use is made of integer approximations to conditional leastsquares transformations. The ambiguity transformation allows one to transform the original DD ambiguities, their least-squares estimates and their corresponding variance-covariance matrix as

$z=Z^{*} a, \hat{z}=Z^{*} \hat{a}, Q_{\hat{z}}=Z^{*} Q_{\hat{a}} Z$

Based on this ambiguity transformation, the sum of squares $e_{2}$ of Eq. (3) can be expressed in terms of the new ambiguities. The corresponding minimization problem can then be formulated as

$\min _{z_{i} \in Z} \sum_{i=1}^{n}\left(\hat{z}_{i \mid I}-z_{i}\right)^{2} / \sigma_{\hat{z}_{i \mid I}}^{2}$

This minimization problem is equivalent to the minimization of $e_{2}$ for $a_{i} \in Z$ if and only if the ambiguity transformation $Z^{*}$ is volume preserving and integer. For a discussion of the properties of $Z^{*}$, also in relation to existing methods of taking linear combinations of DD ambiguities, refer to Teunissen (1995b).

The computation of the integer minimizers $\check{z}_{i}$ of Eq. (5) is performed in the second step of the LAMBDA meth- 
od. Starting from the transformed ambiguity search space

$\sum_{i=1}^{n}\left(\hat{z}_{i \mid I}-z_{i}\right)^{2} / \sigma_{\hat{z}_{i \mid I}}^{2} \leq \chi^{2}$

where $\chi^{2}$ is a suitably chosen positive constant, the solution of Eq. (5) is obtained by means of a search using the following set of bounds

$\left(\hat{z}_{i \mid I}-z_{i}\right)^{2} \leq l_{i} \sigma_{\hat{z}_{i \mid I}}^{2} \chi^{2}$ for $i=1, \ldots, n$

where

$l_{i}=\left(1-\chi_{i-1}^{2} / \chi^{2}\right)$ and $\chi_{i-1}^{2}=\sum_{j=1}^{i-1}\left(\hat{z}_{j \mid J}-z_{j}\right)^{2} / \sigma_{\hat{z}_{j \mid J}}^{2}$

The two sets of inequalities, Eqs. (6) and (7), are equivalent in the sense that they describe the same ambiguity search space. That is, all integer vectors $z \in Z^{n}$ that satisfy Eq. (6) will also satisfy Eq. (7), and vice versa. A search based on the sequential bounds of Eq. (7) will give the desired integer least-squares solution for the transformed ambiguities, $\check{z}_{i}, i=1, \ldots, n$. The search has been described in Teunissen (1995a). Once the integer least-squares vector $\check{z}$ has been found, the corresponding integer least-squares estimates of the original ambiguities can be found by invoking $\check{a}=Z^{*-1} \check{z}$. These integer estimates are then used to compute the 'fixed' baseline solution by means of

$\check{b}=\hat{b} \mid \check{a}=\hat{b}-Q_{\hat{b} \hat{a}} Q_{\hat{a}}^{-1}(\hat{a}-\check{a})$

Alternatively, one can also compute the 'fixed' baseline solution through a direct use of the integer estimate $\check{z}$,

$\check{b}=\hat{b} \mid \check{z}=\hat{b}-Q_{\hat{b} \hat{z}} Q_{\hat{z}}^{-1}(\hat{z}-\check{z})$

The present contribution is organized as follows. In Sect. 2 the size and shape of the ambiguity search space are discussed. In order to be able to start the search, a choice first has to be made regarding the size of the search space. This choice is discussed in Sect. 2.1. In Sect. 2.2 it is shown that the search for the integer leastsquares ambiguities performs poorly when this search takes place in the space of the original DD ambiguities. This poor performance is explained by means of the discontinuity in the spectrum of sequential conditional variances (by 'spectrum' we mean a range of values of a quantity). This discontinuity is a direct consequence of the fact that the observation equations are parametrized in terms of DD ambiguities. Through a decorrelation of the ambiguities, transformed ambiguities can be obtained which will generally have a flat and lower spectrum of conditional variances. The transformed spectra and their relation to the original spectra are the topic of Sect. 3. It is shown what one can typically expect of the transformed spectra when different measurement scenarios are used. The impact on the transformed spectrum of satellite redundancy and the use of dualfrequency phase data is discussed in Sect. 3.1, and the impact of the observation time span and the use of code data is treated in Sect. 3.2. In Sect. 3.3, the statistics of the decorrelated ambiguities are presented. In particular, the dramatic improvement in precision and correlation of the transformed ambiguities is highlighted. Finally in Sect. 4, some issues relevant to different applications of the method are touched upon.

\section{The ambiguity search space}

The decorrelating ambiguity transformation $Z^{*}$ allows for transformation of the ambiguity search space,

$\sum_{i=1}^{n}\left(\hat{a}_{i \mid I}-a_{i}\right)^{2} / \sigma_{\hat{a}_{i \mid I}}^{2} \leq \chi^{2} \stackrel{Z^{*}}{\Rightarrow} \sum_{i=1}^{n}\left(\hat{z}_{i \mid I}-z_{i}\right)^{2} / \sigma_{\hat{z}_{i \mid I}}^{2} \leq \chi^{2}$

These two search spaces contain the same number of integer vectors (grid points), but they differ in shape. The number of grid points contained in them can be controlled by $\chi^{2}$. In Sect. 2.1, ways of choosing suitable values for $\chi^{2}$ are discussed. The difference in shape between the two search spaces is usually very pronounced; in particular, when the data are based on very short observation time spans. The shape of the original DD ambiguity search space is then very elongated, whereas the shape of the transformed search space is closer to that of an $n$-sphere. The shape of the ambiguity search space is governed by the ambiguity variancecovariance matrix. The significance of the variances $\sigma_{\hat{a}_{i \mid}}^{2}$ and $\sigma_{\hat{z}_{i \mid l}}^{2}, i=1, \ldots, n$, in the denominators of the sum of squares already given will be discussed in Sect. 2.2.

\subsection{On the choice of size of the ambiguity search space}

It will be clear that one needs to decide upon a value for the positive constant $\chi^{2}$ before one of the two inequalities in Eq. (10) can actually be used to set up a search. The value chosen for $\chi^{2}$ should not be too small, but also not too large. Too small a value will result in an ambiguity search space which fails to contain the desired integer least-squares solution. In that case we would be trying to solve a problem which fails to have a solution. Too large a value, however, is also not desirable, since it will result in an ambiguity search space having an abundance of grid points. In order to choose a value for $\chi^{2}$, some different lines of thought may be followed. One can differentiate between approaches which make use of the observed data and those that do not. Approaches not making use of the observed data can already be used at the design stage, prior to the actual measurement stage. They allow one to infer whether one can expect to have a successful validation or not.

An appealing approach, from a statistical testing point of view, is to rely on and make use of the statistical properties of the least-squares estimates of the DD ambiguities. These may be either the original estimates $\hat{a}_{i}$, or the transformed estimates $\hat{z}_{i}$. If the least-squares DD ambiguities are normally distributed with mean vector $a$ and variance-covariance matrix $Q_{\hat{a}}$, then the sum of squares of Eq. (10) will have a central Chi-square 
distribution with $n$ degrees of freedom (it will have a central F-distribution if $Q_{\hat{a}}$ has been scaled with the appropriate a posteriori variance factor). As a reference value for $\chi^{2}$ one may now consider choosing the $\alpha$-percentage point of the Chi-square (or F-) distribution. With this choice for $\chi^{2}$, one is of course not certain whether the search will succeed in finding the desired integer least-squares solution. It may still happen that the ambiguity search space is too small and therefore fails to contain a grid point. Although it cannot be guaranteed that the ambiguity search space contains a grid point with this choice, we do know there is a $(1-\alpha)$-percent chance that it will contain one. This grid point will then be the mean of the least-squares ambiguity vector $\hat{a}$, or, if the transformed ambiguities are used, it will be the mean of the least-squares ambiguity vector $\hat{z}$.

In order to be able to choose a value for $\chi^{2}$ which is neither too small nor too large, it is desirable to have a diagnostic quantity which can be used to measure the number of grid points located inside the ambiguity search space. Preferably, such a measure should be easy to compute, without the necessity actually to have to compute all grid points inside the ambiguity search space. Unfortunately, however, it is very difficult in general to find a measure that exactly gives the number of grid points located in the search space. For some simple geometric figures it is possible; for instance, if we consider the $n$-dimensional box centred at a grid point, say the origin $\left(\left|a_{i}\right| \leq 1, i=1, \ldots, n\right)$, then it is not difficult to show that the number of grid points contained by it equals $3^{n}$. For our ambiguity search space however, no such simple expression can be given. We believe though that it is possible to give a fair approximation to the number of the grid points located in the ambiguity search space. Based on our numerical experiments, which were executed in the context of single-baseline determinations, we believe that the volume of the ambiguity search space gives a reasonable indication of the number of grid points. The usage of the volume was already proposed in Teunissen (1993).

In Fig. 1 the volume of the ambiguity search space has been compared to the actual number of grid points inside the search space (candidates). Shown are the results of ten experiments, each experiment based on an observation time span of $1 \mathrm{~s}$ using two epochs of data to seven satellites. The inset in Fig. 1 shows the ratios between the actual number of grid points and the volume.

The figure is based on dual-frequency phase data. The volume chosen for the search space ranges from 1 to 100 , with an increment of one. The lines run under approximately 45 degrees. This shows that the volume is indeed a good indicator for the actual number of integer candidate vectors in the ambiguity search space. See also Teunissen et al. (1996).

The volume of the ambiguity search space is easy and straightforward to compute. We refer to some of the results already computed for the purpose of the sum of squares of Eq. (10). The formula for the volume, $V_{n}$, of the ambiguity search space $(\hat{a}-a)^{*} Q_{\hat{a}}^{-1}(\hat{a}-a) \leq \chi^{2}$ reads (Apostol 1969)

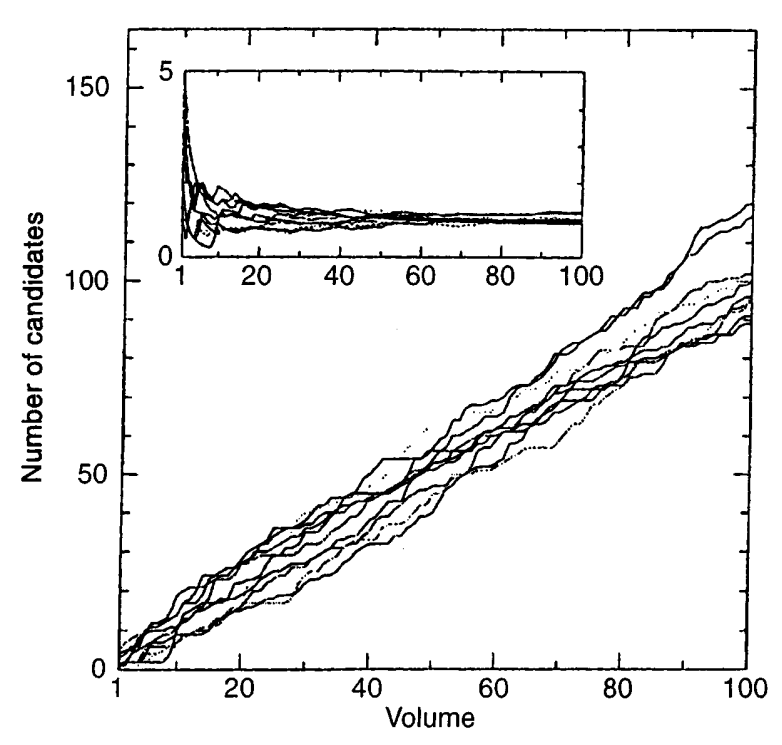

Fig. 1. Number of grid points inside ambiguity search space versus the volume of the search space for dual frequency, phase-only case

$V_{n}=\chi^{n}\left|Q_{\hat{a}}\right|^{1 / 2} U_{n}$

where $\left|Q_{\hat{a}}\right|$ is the determinant of $Q_{\hat{a}}$ and $U_{n}$ is the volume of the unit sphere in $R^{n}$. Using the $\mathrm{LDL}^{*}$-decomposition based sum of squares of Eq. (10), the determinant of $Q_{\hat{a}}$ becomes very easy to compute. It equals the product of all ambiguity variances in the spectrum of conditional variances. Hence,

$\left|Q_{\hat{a}}\right|=\prod_{i=1}^{n} \sigma_{\hat{a}_{i \mid I}}^{2}$

Note that since both ambiguity search spaces, the original and the transformed one, have the same volume, we have $\left|Q_{\hat{a}}\right|=\left|Q_{\hat{z}}\right|$. Hence, Eq. (12) can also be computed from the conditional variances of the transformed ambiguities. In order to compute Eq. (11), we still need to compute $U_{n}$. The volume of the unit sphere is given as

$U_{n}=\pi^{n / 2} / \Gamma(n / 2+1)$, with $\Gamma(x)=\int_{0}^{\infty} e^{-t} t^{x-1} \mathrm{~d} t, x>0$

where $\Gamma(x)$ is the gamma-function. For our purposes it is not necessary to evaluate the preceding integral explicitly. Making use of the recurrence relation $\Gamma(x+1)=x \Gamma(x)$, for $x>0$, and the fact that $\Gamma(1 / 2)=\sqrt{\pi}$ and $\Gamma(1)=1$, it follows for even and odd $n$, respectively, that

$$
\begin{aligned}
& \Gamma(n / 2+1)=(n / 2) !, n=0,2,4, \ldots(0 !=1) \\
& \Gamma(n / 2+1)=\frac{n}{2} \frac{n-2}{2} \cdots \frac{1}{2} \sqrt{\pi}, n=1,3,5, \ldots
\end{aligned}
$$

The choice for the size of the ambiguity search space can now be made as follows. Depending on the approximate number of grid points required, the value of the volume $V_{n}$ is set. Compute the volume $U_{n}$ of the unit 
sphere by means of Eq. (13) and (14), and use the conditional variances to compute the determinant $\left|Q_{\hat{a}}\right|=\left|Q_{\hat{z}}\right|$ by means of Eq. (12). Then, using $V_{n}, U_{n}$ and $\left|Q_{\hat{a}}\right|$, the value for $\chi^{2}$ follows from Eq. (11). Of course, since $V_{n}$ is only an indicator of the number of grid points, it is not so much the precise value of $V_{n}$ that counts, but more its order of magnitude.

An important additional use that can be made of the volume $V_{n}$ lies in the area of validating the integer ambiguities. Once $\chi^{2}$ has been computed based on a chosen value for $V_{n}$, one can use the Chi-square (or F-) distribution to find the corresponding $\alpha$-percentage point. If it then turns out that $\alpha$ is too large, one may conclude that validation of the integer ambiguities will become problematic. This approach was used in the analytical study in Teunissen (1996). The advantage of using the volume $V_{n}$ in this context of validation is that it can already be used at the designing stage to infer whether one is likely to have a successful validation or not.

Finally, an alternative approach to set the value of $\chi^{2}$ at an appropriate level for the search is one that makes use of the observed data. Hence it cannot be used at the design stage. The idea is simply the following. Round each of the entries of $\hat{a}$ to their nearest integer, substitute the so-obtained integer vector for $a$ into the first sum of squares of Eq. (10) and then take $\chi^{2}$ to be equal to the value of the sum of squares. This approach ensures that the ambiguity search space will contain at least one grid point. Of course, there is a possible disadvantage to this approach, namely that the value for $\chi^{2}$ may be overly conservative. This will especially be the case when the ambiguities are highly correlated and their ambiguity search space is highly elongated, as is the case when DD ambiguities are used based on short observation time span data. Hence one should not use this approach for the original DD ambiguity search space. It works much better, however, when used for the transformed ambiguity search space. In fact, for validation purposes where usually the most likely and second most likely integer vector is needed, the same idea can be used to set $\chi^{2}$ to a value that guarantees that a minimum of two grid points are located inside the search space. In this case, one grid point is obtained by rounding all entries of $\hat{z}$ to their nearest integer and another grid point is obtained by rounding one of the entries of $\hat{z}$ to its second nearest integer, while the remaining entries are rounded to their first nearest integer. The value of $\chi^{2}$ is then taken to be equal to the larger of the two values the sum of squares of Eq. (10) takes for the two grid points. The reason why this approach works for the transformed ambiguities is, as will be shown in Sect. 3.3, due to the following two properties. Firstly, the shape of the transformed ambiguity search space is much closer to that of an $n$-sphere than the shape of the original DD search space. Secondly, the individual transformed ambiguities are also much more precise than the original ones.

Figure 2 shows two cases where the volume of the ambiguity search space has been computed following the preceding principle. The first case holds for the transformed ambiguity search space, whereas the second holds for the original ambiguity search space. From the
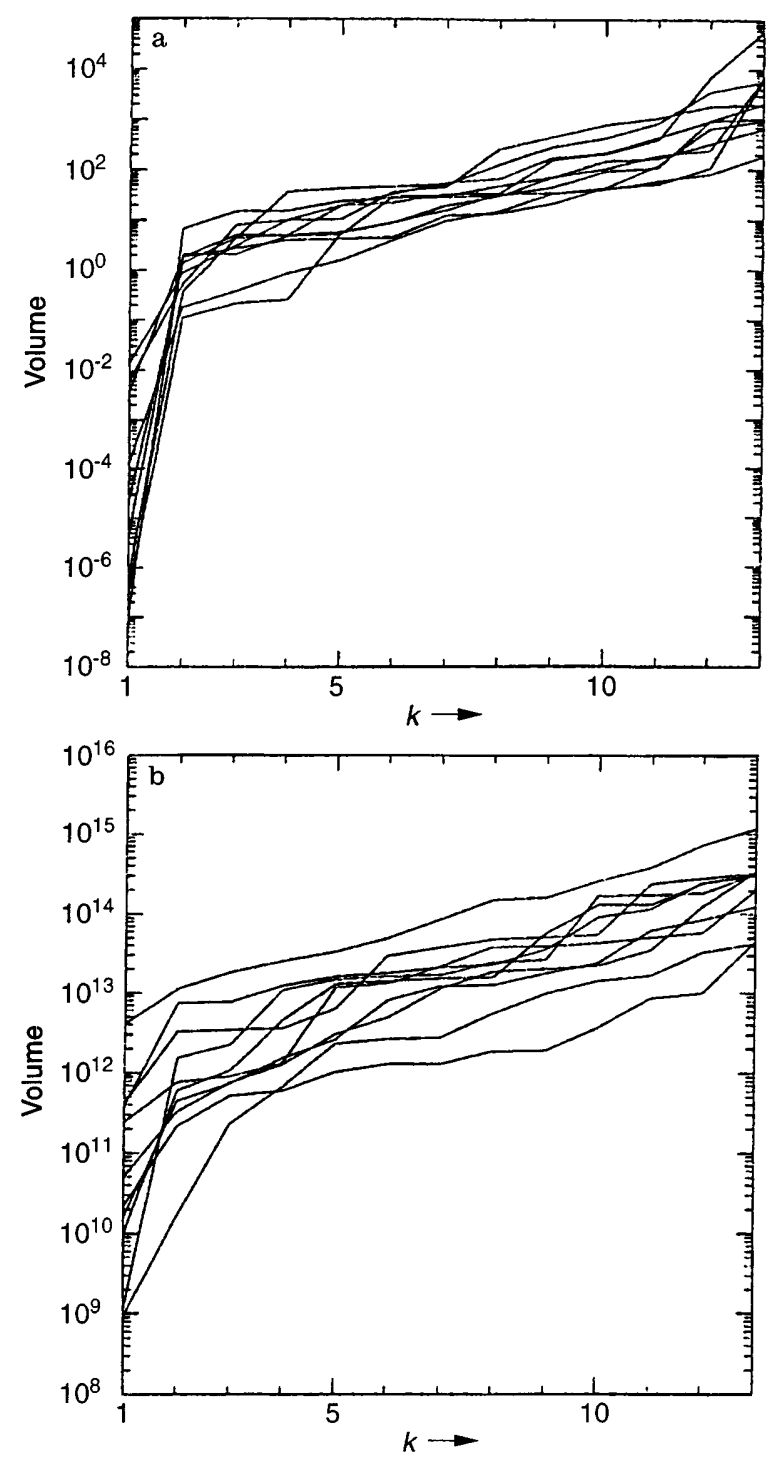

Fig. 2a, b. Volume of $\mathbf{a}$ transformed and $\mathbf{b}$ original DD ambiguity search space for $k=1, \ldots,(n+1)$ rounded integer vectors

real-valued least-squares estimate of the transformed ambiguities, $\hat{z}$, the following $(n+1)$ integer vectors were obtained: the integer vector which has as its entries the integers nearest to the corresponding entries of $\hat{z}$, and the $n$ integer vectors one gets when rounding all entries of $\hat{z}$ but one to the nearest integer; the remaining entry being rounded to the next nearest integer. Each of these $(n+1)$ integer vectors were substituted for $z$ into the second sum of squares of Eq. (10) and the corresponding value of the sum of squares was then used to compute the $(n+1)$ volume values.

Thus we now have the volumes of $(n+1)$ transformed ambiguity search spaces of which it is guaranteed that each one contains minimally one grid point. The sequence of $(n+1)$ volumes - sorted in increasing order - are shown for ten experiments in Fig. 2a. The data used for these ten experiments are the same as used for Fig. 1. Figure 2a shows that the second smallest volume - the one that guarantees at least two integer candidates - is 
smaller than ten. This, together with the results of Fig. 1, shows that the actual number of grid points inside this search space is less than twenty. Hence, we have indeed been able to set the size of the ambiguity search space at not too a high level, while at the same time making sure that the search space contains at least two grid points. Figure $2 \mathrm{~b}$ shows the case where the same procedure was followed, but now for the original ambiguity search space. It will be clear from these results that in this case the procedure has no chance of success whatsoever. With the original DD ambiguities, the volume values become too large for a reasonable search space.

\subsection{On the discontinuity in the DD spectrum}

The elongated shape of the DD ambiguity search space is also a hindrance for the computation of the integer least-squares estimates of the DD ambiguities. In the introduction, we already mentioned that the bottleneck in solving the integer least-squares problem, Eq. (2), lies in the difficulty one has in efficiently solving the minimization problem

$\min _{a_{i} \in Z} \sum_{i=1}^{n}\left(\hat{a}_{i \mid I}-a_{i}\right)^{2} / \sigma_{\hat{a}_{i \mid I}}^{2}$

In order to understand this difficulty properly, we will show what happens when Eq. (15) is solved in the same way as we proposed to solve its transformed expression, Eq. (5). Therefore, in analogy with Eq. (6), we start from the DD ambiguity search space

$\sum_{i=1}^{n}\left(\hat{a}_{i \mid I}-a_{i}\right)^{2} / \sigma_{\hat{a}_{i \mid I}}^{2} \leq \chi^{2}$

and use, in analogy with Eq. (7), the following $n$-number of search bounds

$$
\left\{\begin{array}{c}
\left(\hat{a}_{1}-a_{1}\right)^{2} \leq \sigma_{\hat{a}_{1}}^{2} \chi^{2} \\
\left(\hat{a}_{2 \mid 1}-a_{2}\right)^{2} \leq \sigma_{\hat{a}_{2 \mid 1}}^{2} \chi^{2}\left(1-\left(\hat{a}_{1}-a_{1}\right)^{2} / \sigma_{\hat{a}_{1}}^{2} \chi^{2}\right) \\
\cdot \\
\cdot \\
\left(\hat{a}_{n \mid N}-a_{n}\right)^{2} \leq \sigma_{\hat{a}_{n \mid N}}^{2} \chi^{2}\left(1-\sum_{j=1}^{n-1}\left(\hat{a}_{j \mid J}-a_{j}\right)^{2} / \sigma_{\hat{a}_{j \mid J}}^{2} \chi^{2}\right)
\end{array}\right.
$$

Now, consider the number of integer candidate vectors that progressively satisfy the given bounds. For that purpose, we introduce the concept of the $j$-level ambiguity search space or the partial ambiguity search space of level $j$ which is defined as

$\sum_{i=1}^{j}\left(\hat{a}_{i \mid I}-a_{i}\right)^{2} / \sigma_{\hat{a}_{i \mid I}}^{2} \leq \chi^{2}$

Note that the integer minimizers of the left side of this inequality are identical to the integer minimizers of
Eq. (15), provided that the last $(n-j)$ ambiguities in Eq. (15) are relaxed to be real numbers instead of integers. Also note that the partial search space Eq. (18) is described by the first $j$ bounds in Eq. (17). For our purpose, it is now of interest to consider the number of integer vectors that satisfy the inequality of Eq. (18), when the level $j$ is changed from 1 up to and including $n$. The relevance of this information is that it gives an indication on how well the search for the integer leastsquares solution will perform. For instance, if the number of integer candidate vectors decreased as the level $j$ increases, then, for a number of integer candidate vectors $\left(a_{1}, \ldots, a_{j}\right)^{*}$ at level $j$, no integer $a_{j+1}$ could be found such that $\left(a_{1}, \ldots, a_{j}, a_{j+1}\right)^{*}$ is an integer candidate vector at level $j+1$.

To illustrate the situation, a typical GPS example was taken, based on dual-frequency data for a single baseline using carrier phases only. Seven satellites were observed over an observational time span of only $1 \mathrm{~s}$ (two epochs of observation), with the a priori standard deviation of the $L_{1}$ and $L_{2}$ carrier phases set at $\sigma=3 \mathrm{~mm}$ and the constant $\chi^{2}$ chosen equal to 100 . The dashed curve of Fig. 3 shows the number of integer candidates as function of the level $j$. The number of integer candidates can be read from the vertical axis at the left. Note its logarithmic scale. We observe that the dashed curve increases from level 1 , reaches its maximum at level 3 , and from then on strictly decreases. It finally reaches the value of 966, which equals the number of integer vectors (or grid points) that are located inside the ambiguity search space of Eq. (16). The behaviour shown here is highly typical and prevails when one works with GPS DD ambiguities which are based on very short observation time span data. A direct consequence of the sharp decrease in function value when going from level 3 to level 4 , is that the search for the integer least-squares solution of the DD ambiguities will suffer from a high likelihood of halting. By halting we mean that the search cannot be continued, as no integer satisfies the bounds of Eq. (17) for the next level.

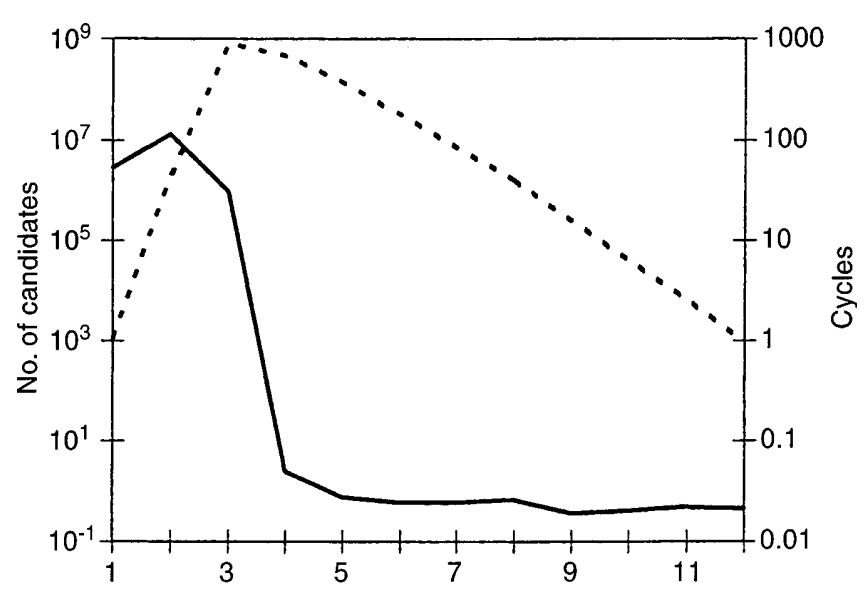

Fig. 3. Number of integer candidate vectors per level $\mathrm{j}$ (dashed curve) and the corresponding spectrum of DD ambiguity conditional standard deviations in cycles (full curve) 
In order to understand the reason for this inefficiency, we need to understand why the dashed curve of Fig. 3 exhibits this typical behaviour. Consider therefore the individual bounds of Eq. (17). Each one of these bounds generally admits less integer candidates the tighter the bounds are. It follows from Eq. (17) that these bounds depend, apart from the constant $\chi^{2}$, on the conditional least-squares ambiguity estimates $\hat{a}_{i \mid I}$ and their variances $\sigma_{\hat{a}_{i \mid}^{2}}^{2}$.

The bounds already have the natural tendency of getting tighter with increasing level. For instance, $\left(1-\sum_{i=1}^{j}\left(\hat{a}_{i \mid I}-a_{i}\right)^{2} / \sigma_{\hat{a}_{||}}^{2} \chi^{2}\right)$ will always get smaller as $j$ increases. Also, when we assume that all unconditional variances are of the same order, the sequential conditional variances tend to get smaller as more constraints are imposed. This tendency is however inherent to the use of the sequential conditional least-squares based bounds of Eq. (17) and does not explain why the dashed curve of Fig. 3 shows such a clear peak. Since the greater behaviour of the dashed curve in Fig. 3 is typical for all singlebaseline solutions, it has to be due to the model-driven, GPS-based characteristics of the sequential conditional variances Teunissen et al. (1994). For the same configuration, we computed the sequential conditional standard deviations of the twelve ambiguities, shown in Fig. 3 as the full curve. The values taken by the conditional standard deviations can be read from the vertical axis on the right. Again note the logarithmic scale.

The shape of the curves in Fig. 3, now allows us to explain why the number of integer candidate vectors increases from level 1 to level 3 and why it decreases when level 3 is passed. The figure shows three very large conditional standard deviations at the beginning and then nine extremely small ones. The discontinuity corresponds with the peak of the dashed curve. Because of the fact that the first three conditional standard deviations are large, the first three bounds of Eq. (17) will be rather loose. Hence, the first bound of Eq. (17) already admits a very large number of integer candidates and this number progressively increases until one hits level four. After the third level, a sudden dramatic drop in value of the conditional standard deviation is experienced. The fourth and remaining conditional standard deviations are all of about the same small order. Because of this tremendous drop in value, the fourth and remaining bounds of Eq. (17) will all be very tight indeed. Hence, of the many integer candidate vectors that satisfied the first three bounds, a large number will not pass the test of the fourth and remaining bounds. As a consequence, search halting is experienced.

The shape of the curves shown in Fig. 3 is typical for GPS short-baseline positioning and can be explained as follows. Assume that three out of the $n$ DD ambiguities are known. The three DD phase observation equations in which these three ambiguities appear then only have left the three baseline components as unknowns. Hence, these three equations form a system of three equations in three unknowns. This system is uniquely solvable, assuming that configuration defects in the corresponding relative receiver-satellite geometry are absent. Due to the high precision of the three DD carrier-phase observables, the three baseline components are then determined with a correspondingly high precision. Now consider, for the same observation epoch, the remaining $(n-3)$ DD phase observation equations. This set of equations again constitutes a uniquely solvable system, but now of $(n-3)$ equations with $(n-3)$ unknowns. The unknowns are the remaining $(n-3)$ ambiguities and the 'observations' are linear combinations of the remaining $(n-3)$ DD carrier phases and of the baseline components determined from our earlier set of three equations. Since these 'observations' can be seen as being linear combinations of all $n$ DD carrier phases, it follows that the remaining $(n-3)$ DD ambiguities can be determined with a very high precision indeed. The conclusion is thus reached that the ambiguities can be determined with a very high precision once three out of the $n$ ambiguities are known. This is precisely what is seen in the shape of the spectrum of conditional variances. For instance, once we arrive at the fourth conditional variance, the precision is considered of an ambiguity which is conditioned on knowing the first three ambiguities. Note that the use in Hatch (1991) of two groups of satellites, a primary group and a secondary group, is essentially based on a recognition of the discontinuity in the spectrum of conditional variances already explained.

\section{The spectra of the decorrelated ambiguities}

It is important to understand that the discontinuity in the spectrum of conditional variances is due to the fact that the DD phase observation equations have been parametrized in terms of one particular set of ambiguities, namely the DD ambiguities. With the DD ambiguities, one and only one ambiguity is assigned as unknown parameter per observation equation. It is this assignment which makes it possible to determine the three-dimensional baseline with such a high precision once only three ambiguities are assumed known. This would generally not have been possible if more than one ambiguity was assigned to the phase observation equation. For instance, if we used a full ambiguity transformation matrix $Z^{*-1}$ to reparametrize the DD ambiguity vector $a$, by substituting $a=Z^{*-1} z$ into the system of DD phase observation equations, then generally no three ambiguities from $z$ could be found, which would allow one to determine the baseline once these ambiguities are assumed known. Thus, the shape of the spectrum of the new ambiguities will generally be quite different from the shape as seen in the spectrum of the DD ambiguities. This therefore gives the opportunity to search for an ambiguity transformation which allows one to mould the spectrum in an appropriate shape. As discussed in Teunissen (1993, 1995a), this is precisely what the ambiguity transformation does when it aims at a decorrelation of ambiguities, thereby removing the large differences between the conditional variances of the ambiguities. 
In this section results will be presented that can typically be achieved through the decorrelation of the ambiguities. The transformed spectra will be shown and compared to the original spectra. The flattening and lowering of the spectra will be discussed and the impact of different measurement scenarios on the shape of the transformed spectra will be explained. In particular, two sets of cases will be treated. The first set is discussed in Sect. 3.1 and considers the impact of satellite redundancy and the use of dual-frequency phase. The second set is discussed in Sect. 3.2 and considers the impact of the observation time span and the use of code (pseudorange) data.

\subsection{The impact of satellite redundancy and dual-frequency phase}

In this section the impact of satellite redundancy and the use of dual-frequency phase data on the original and transformed spectrum of conditional variances is considered. It is well known that the use of more than four satellites and the use of a second frequency has a very favourable influence on the ability to resolve the integer ambiguities. In general, this can be understood by referring to the increasing redundancy. For instance, if $(n+1)$ satellites are observed then the redundancy equals $(n-3)$ if two epochs of only single-frequency phase data are used. This shows that every satellite which is added to the minimum number four increases the redundancy by one. In case of two epochs with dualfrequency data, the redundancy equals $(2 n-3)$. In this case, the redundancy is increased by two for every satellite which is added.

An increase in redundancy implies a 'stronger' model, thereby allowing one to determine the unknown parameters with an improved precision. This, however, is a general statement, and for our GPS applications is too imprecise to enable us really to understand the impact of redundancy. We shall therefore consider the spectrum of conditional variances for both the original and transformed ambiguities. In particular the beneficial role of redundancy for the level of the transformed spectrum will be highlighted. Four cases will be considered. They are

a. single frequency $\left(L_{1}\right)$, no satellite redundancy $(4 s v)$,

b. single frequency $\left(L_{1}\right)$, with satellite redundancy $(7 s v)$,

c. dual frequency $\left(L_{1} / L_{2}\right)$, no satellite redundancy $(4 s v)$,

d. dual frequency $\left(L_{1} / L_{2}\right)$, with satellite redundancy $(7 s v)$.

The original and transformed spectra of these four cases are shown in Fig. 4. These spectra are based on an observation time span of only $1 \mathrm{~s}$ (two epochs of observation), with the a priori standard deviation of the carrier phases set at $\sigma=3 \mathrm{~mm}$. Only phase data were used. Since we know that the determinant of the original ambiguity variance-covariance matrix equals that of the transformed variance-covariance matrix, and also that
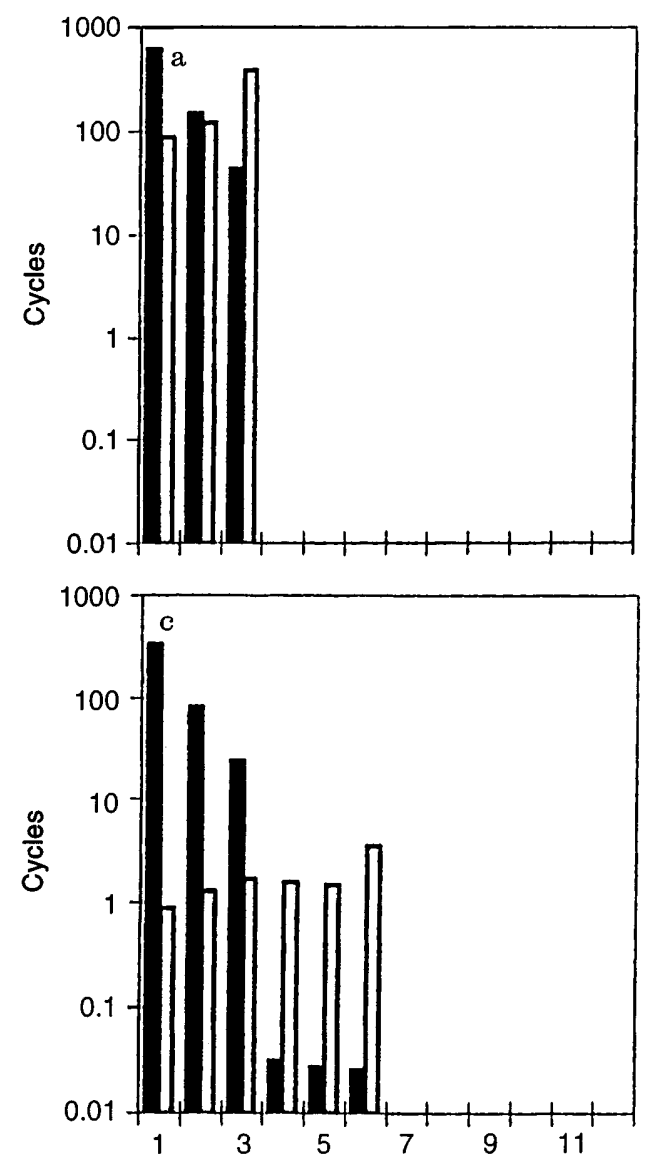
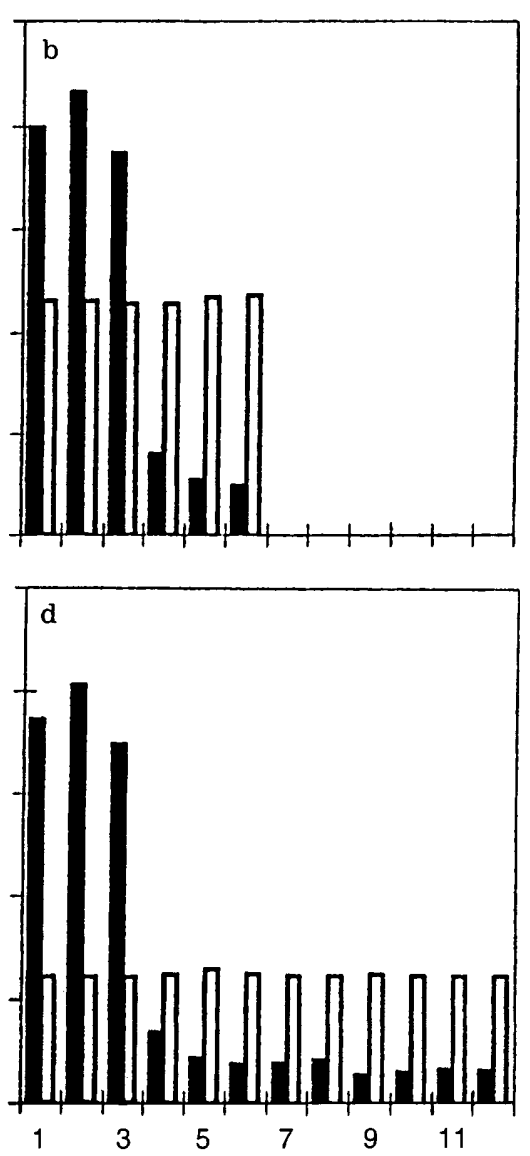

Fig. 4a-d. Original and transformed spectra of conditional standard deviations in cycles. The sequential conditional standard deviations of the original DD ambiguities, $\sigma_{\hat{a}_{i \mid l}}$, are shown as black bars and their counterparts in the transformed spectrum, $\sigma_{\hat{z}_{i l l}}$, are shown as white bars. a no redundancy, $\mathbf{b}$ satellite redundancy, c dual frequency, $\mathbf{d}$ satellite redundancy and dual frequency 
the determinant of the variance-covariance matrix equals the product of all sequential conditional variances, we have the equality

$$
\prod_{i=1}^{n} \sigma_{\hat{a}_{i \mid I}}=\prod_{i=1}^{n} \sigma_{\hat{z}_{i \mid I}} \quad(\text { cycles } n)
$$

Thus we have as a rule, because of the logarithmic scale along the vertical axis, that the sum of the areas of the black bars equals the sum of the areas of the white bars.

Let us now start our discussion of Fig. 4. Part a is based on single-frequency data and four satellites. Hence there are three ambiguities and redundancy is absent. Due to the absence of redundancy, all three conditional standard deviations of both spectra are quite large. The values of the conditional standard deviations of the transformed ambiguities start at a much lower level and increase, instead of decrease.

When three satellites are added to the configuration, we obtain Fig. 4b. The number of ambiguities has increased from three to six. The inclusion of satellite redundancy has two effects on the spectrum of DD ambiguities. First we note a general improvement in precision of the original three DD ambiguities. The first conditional standard deviation, $\sigma_{\hat{a}_{i \mid !}}$ for $i=1$, is actually an unconditional standard deviation and its value is representative for the precision of the DD ambiguities. Its value is significantly smaller than its counterpart of Fig. 4a. The amount by which the precision of the original three DD ambiguities improves depends on the relative receiver-satellite geometry of the seven-satellite configuration. For instance, the improvement will be large when the additional three satellites are close to the original four satellites. A second consequences of including three additional satellites is that the spectrum now contains three conditional standard deviations that are very much smaller than the first three. This is the discontinuity in the spectrum, which was explained earlier in Sect. 2.2. It is the presence of this discontinuity and thereby the presence of satellite redundancy, that has a very favourable influence on the transformed spectrum. Note, that the transformed spectrum is quite flat and that its level is dramatically lower than the large conditional standard deviations of the original spectrum. The conditional standard deviations of the transformed ambiguities are all in the order of a few cycles. The low level of the transformed spectrum can be explained as follows. By aiming at decorrelation, the ambiguity transformation $Z^{*}$ tries to lower the large conditional standard deviations. However, since the condition Eq. (19) holds true, large conditional standard deviations in the spectrum can only be lowered significantly if the spectrum already contains some very small conditional standard deviations. We thus see that it is the presence of the three very small conditional standard deviations in the spectrum of Fig. $4 \mathrm{~b}$ that allows us to push the three large ones to a much lower level.

When the second frequency $L_{2}$ is added to the configuration, which formed the basis of Fig. 4a, we obtain Fig. 4c. The number of ambiguities has now been doubled from three to six. Quite similar to the case when satellite redundancy was added, two effects can be seen on the spectrum of DD ambiguities. The precision of the DD ambiguities has improved and again a discontinuity in the spectrum appears. Note that the signature of the first three DD conditional standard deviations of Fig. 4c is identical to the one of Fig. 4a. This is of course due to the fact that both cases are based on an identical relative-satellite geometry. The presence of the three very small conditional standard deviations again allows us to flatten and lower the spectrum. The conditional standard deviations of the transformed ambiguities are now of the order of a few cycles.

When both dual frequency and satellite redundancy are used we obtain Fig. 4d. The number of ambiguities now equals twelve. The signature of the first six DD conditional standard deviations is identical to that of Fig. 4b. This is due to the fact that both cases are based on the same relative receiver-satellite geometry. The precision of the ambiguities has improved however, due to the additional redundancy. Again we note a large drop in value in the original spectrum when moving from the third to the fourth conditional standard deviation. In the present case however, we now have, when compared to cases $\mathrm{b}$ and $\mathrm{c}$ of Fig. 4, six additional very small conditional standard deviations. This therefore allows us to push down the level of the transformed spectrum even further. The conditional standard deviations of the transformed ambiguities are now at the very low level of a few tenths of a cycle.

\subsection{The impact of the observation time span and code data}

In the previous section the relevance for the transformed spectrum, of satellite redundancy and of using dualfrequency data has been shown. Through this type of redundancy, we obtain very small conditional variances, which allows us to bring the transformed spectrum to a dramatically lower level than the original spectrum. As a result, the efficiency in the search for the integer leastsquares solution is greatly enhanced. There are of course other means of improving the precision of the ambiguities. In this section we will therefore show what the impact is of the observation time span and of the use of code (pseudo-range) data. We will also consider the effect of using widelaning. The idea behind the use of widelane ambiguities is in some sense similar to the idea of using the ambiguity transformation $Z^{*}$, see Teunissen (1995b). Four cases will be considered; they are all based on the use of dual-frequency phase, observing seven satellites, using only two epochs of observation. The four cases are

a. $\Delta t=1 \mathrm{~s}$, phase only,

b. $\Delta t=60 \mathrm{~s}$, phase only,

c. $\Delta t=1 \mathrm{~s}$, code included,

d. $\Delta t=1 \mathrm{~s}$, phase only, but widelaning.

The first case is shown in Fig. 5a. It is identical to Fig. $4 \mathrm{~d}$ and will be used as our reference. Figure $5 \mathrm{~b}$ shows the situation when the observation time span 

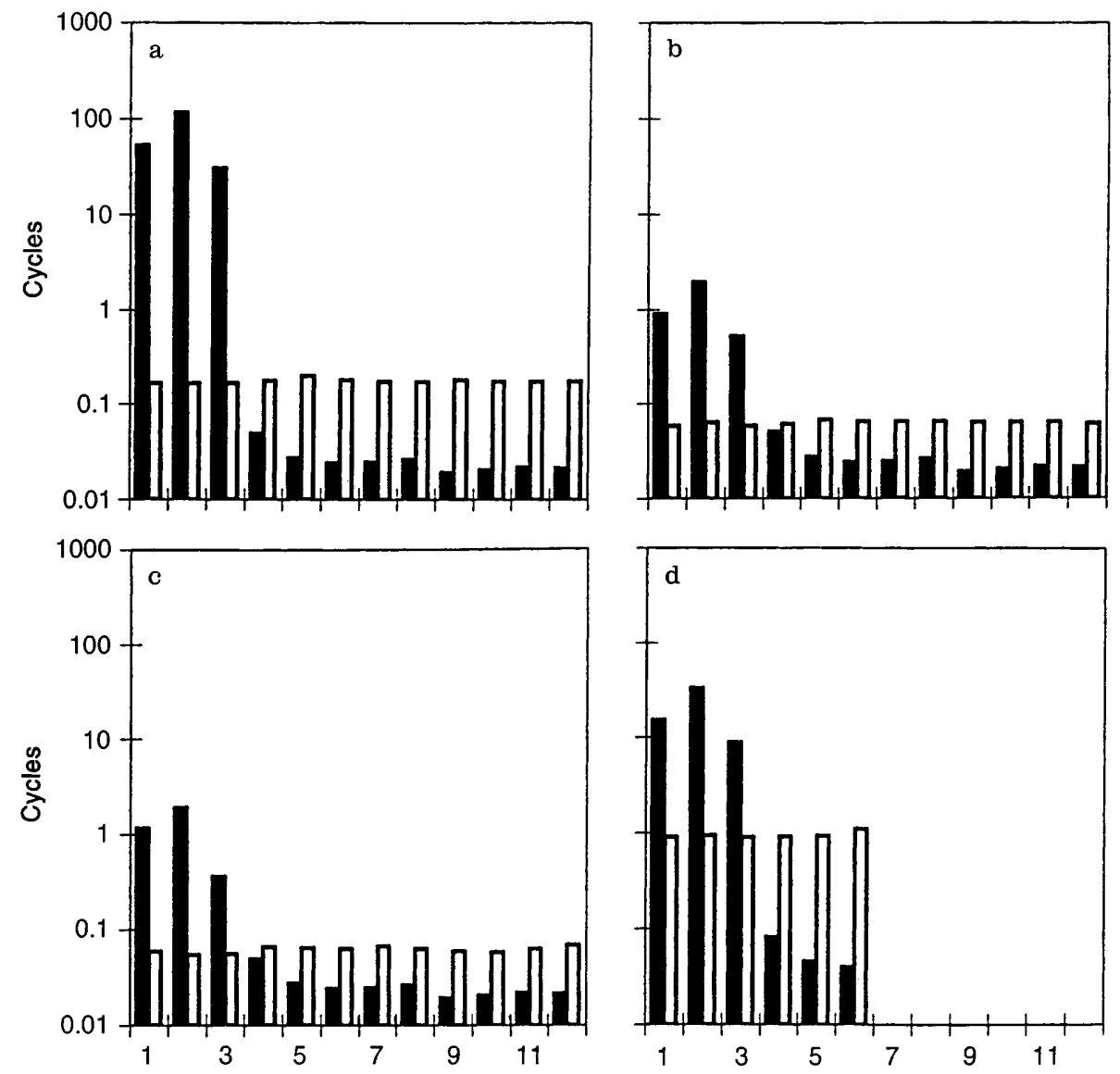

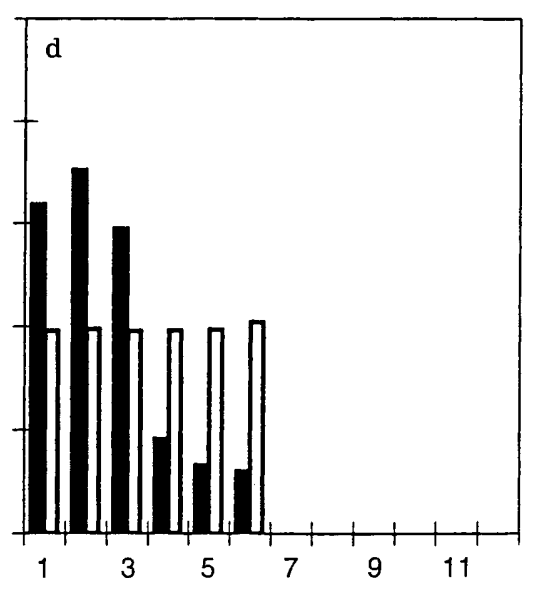

Fig. 5a-d. Original and transformed spectra of conditional standard deviations in cycles. a $L_{1} / L_{2}$ phase, $\Delta t=1 \mathrm{~s}$; b $L_{1} / L_{2}$ phase, $\Delta t=60 \mathrm{~s}$; $L_{1} / L_{2}$ phase + code, $\Delta t=1 \mathrm{~s} ; \mathbf{d}$ widelane phase, $\Delta t=1 \mathrm{~s}$ equals $60 \mathrm{~s}$ instead of only $1 \mathrm{~s}$. Since only two epochs of observation are used, the redundancy for this case is identical to that of Fig. 5a. The two cases differ however in their relative receiver-satellite geometry. The discontinuity in the DD spectrum of Fig. 5b is still present, but it is already somewhat smaller than that of Fig. 5a. When compared to the DD spectrum of Fig. 5a, the three large conditional standard deviations of the DD spectrum of Fig. $5 \mathrm{~b}$ have all been pushed to lower values. The smaller discontinuity in the DD spectrum implies that the search will be less likely to halt. All the same, a significant improvement can be reached by means of the transformed spectrum. The discontinuity has disappeared and the transformed conditional standard deviations are now all less than 0.1 cycle. This level is also lower than the level of the transformed spectrum of Fig. 5a.

In the DD spectrum of Fig. 5b, the first three conditional standard deviations are smaller than their counterparts of Fig. 5a. Note, however, that the nine very small conditional standard deviations have hardly changed at all. We thus see that an extension of the observational time span has a major effect on the first three conditional standard deviations, but only a small effect on the remaining nine conditional standard deviations. In some way this must have to do with the relative receiver-satellite geometry. In order to understand this properly, one needs to make a clear distinction between, on the one hand, the instantaneous receiver- satellite geometry at one single epoch and, on the other, the change in receiver-satellite geometry from epoch to epoch. The precision with which the ambiguities can be estimated is very much dependent on the amount of change that takes place in the receiver-satellite geometry. In fact, least-squares ambiguity estimation, based on phase data only, is impossible when only one epoch of observation is used. Thus, the ambiguity precision is poor when the change in geometry is small, and precision improves the larger the change. This explains why the first three conditional standard deviations of the DD spectrum of Fig. $5 \mathrm{~b}$ are smaller than their counterparts in Fig. 5a. The situation for the remaining nine conditional standard deviations is quite different however. In this case, it is not so much the change in geometry that counts, but the instantaneous geometry itself. Remember from our earlier discussion that once three DD ambiguities are known, the remaining DD ambiguities can be estimated with a very high precision, using only one epoch of phase data. The instantaneous receiversatellite geometries of the two cases, (a) and (b), do not differ significantly, neither do their nine small DD conditional standard deviations.

If dual-frequency code data are added to the dualfrequency phase data of Fig. 5a, Fig. 5c is obtained. The standard deviation of the code observations was set at $\sigma=60 \mathrm{~cm}$. Note that a significant improvement can be obtained through the transformed spectrum. Also note that the spectra of Fig. $5 \mathrm{c}$ resemble those of Fig. 5b. The 
explanation differs however. The first three DD conditional standard deviations of Fig. $5 \mathrm{~b}$ are smaller than their counterparts of Fig. 5a, because of the fact that they are based on a more significant change in receiversatellite geometry. In the case of Fig. 5c however, it is not the change in geometry that counts, but the combination of the instantaneous geometry and the precision of the code data. Using code data of higher precision would lower the values of the first three DD conditional standard deviations. Also the level of the transformed spectrum would then be lower. If in the limiting case one worked with perfectly known code data, then all conditional standard deviations would be of the order of the precision of phase divided by the wavelength.

The data used for Fig. 5d, is identical to the data used for Fig. 5a. In case of Fig. 5d however, the dual-frequency phase data were used to form the so-called widelane linear combinations, see Melbourne (1985), Blewitt (1989), Dong and Bock (1989), Wübbena (1991), Euler and Landau (1992), Cocard and Geiger (1992), Goad (1992) and Teunissen (1995b). As a result, six widelane ambiguities are obtained, instead of the twelve original DD ambiguities. The common rationale behind using the widelane combination is that it has a longer wavelength. One can therefore expect that the widelane ambiguity can be estimated with a precision better than that of the original DD ambiguities. This is also reflected in the widelane spectrum of Fig. 5d. Of course, the widelane ambiguities can also be transformed by means of a decorrelating ambiguity transformation. In the analytical study of Teunissen (1996), it is shown that the widelane ambiguities often appear in the first step when constructing the decorrelating ambiguity transformation $Z^{*}$. The transformed conditional standard deviations are shown in Fig. 5d and they are all of the order of 1 cycle. Note, that they are all clearly larger than the transformed conditional standard deviations of Fig. 5a. This is caused by the fact that in the widelane case only three small conditional standard deviations can be used to pull the larger values down. We may therefore conclude that no advantage is gained from working with the widelane ambiguities. Transforming the original $L_{1} / L_{2}$ DD dual-frequency ambiguities gives a better result.
In order to show that the use of the transformed ambiguities indeed allows one to improve significantly on the search for the integer ambiguities, two examples are given, each showing the number of grid points per partial search space. The two examples are based on the two preceding cases (a) and (c). Figure 6a shows the dual-frequency, phase-only case and Fig. $6 \mathrm{~b}$ the dualfrequency case with code data included. For both cases, the same level of significance was used to set the size of the ambiguity search space. In both cases we clearly see the peak at level 3 (dashed curve), when the original DD ambiguities are used. This peak is completely absent, however, when the transformed ambiguities are used (full curve). Due to the much higher precision of the transformed ambiguities, this curve starts from a lower value and gradually increases (Fig. 6a) or stays constant (Fig. 6b). Per case, the dashed and full curve meet of course at level $n$, because although the original and transformed ambiguity search spaces differ in shape, they still contain the same number of grid points.

\subsection{The statistics of the decorrelated ambiguities}

In this section we will discuss the precision, $\sigma_{\hat{a}}$ and $\sigma_{\hat{z}}$, the elongation, $e_{\hat{a}}$ and $e_{\hat{z}}$, and the decorrelation, $r_{\hat{a}}$ and $r_{\hat{z}}$, of both the original and transformed ambiguities. Results will be given for all seven cases treated in the previous two sections. Elongation is a dimensionless quantity which measures the shape of the ambiguity search space. It equals the square root of the condition number of the ambiguity variance-covariance matrix. Hence, it equals the square root of the ratio of the largest and the smallest eigenvalue of the ambiguity variance-covariance matrix. Elongation is at its minimum when it equals one. In that case, the ambiguity search space becomes a perfect $n$-sphere. Statistically, the elongation can be interpreted as the ratio of two standard deviations; the standard deviation of the linear function of ambiguities having poorest precision, divided by the standard deviation of the linear function of ambiguities having the best possible precision.

In two dimensions, decorrelation is measured by the square root of $r^{2}=1-\rho^{2}$, with $\rho$ being the dimen-
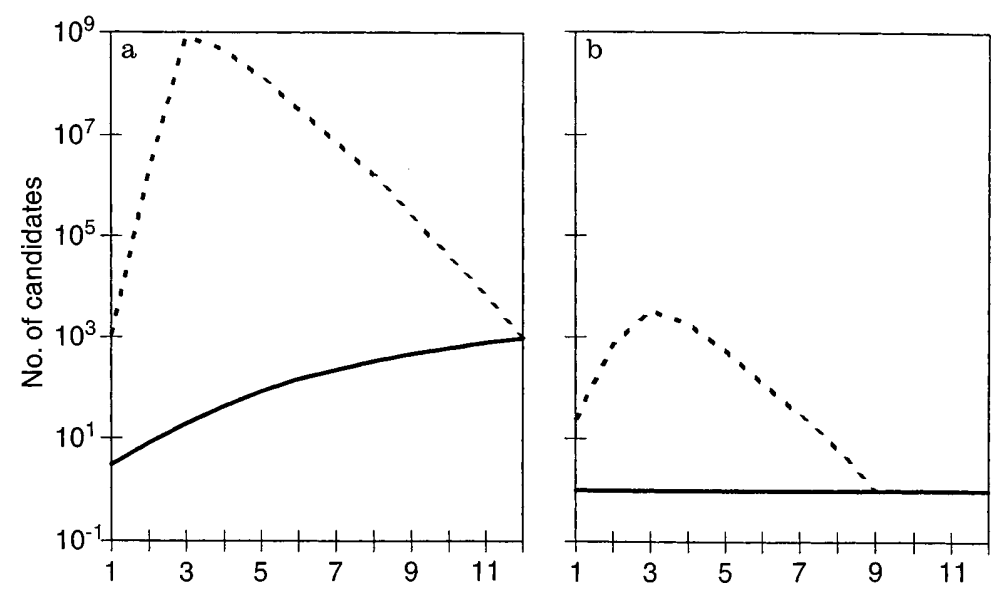

Fig. 6a, b. Number of integer candidate vectors per level $j$, for the original DD ambiguities (dashed curve) and the transformed ambiguities (full curve). a $L_{1} / L_{2}$ phase only, b $L_{1} / L_{2}$ phase + code 
sionless correlation coefficient. Since $1-\rho^{2}$ equals the determinant of the ambiguity correlation matrix $R,|R|$, the decorrelation will be measured in dimensions higher than two, by the square root of the determinant. A full decorrelation corresponds then to $r=1$ and a poor decorrelation with a value close to zero. Geometrically, the decorrelation number $r$ can be given the following useful interpretation. Since $|R|$ equals the determinant of the variance-covariance matrix, divided by the product of variances, $r$ equals the ratio of the volumes of two $n$ boxes. The numerator of this ratio equals the volume of the $n$-box that encloses the ambiguity search space and which has its sides parallel to the principal axes of the search space. The denominator equals the volume of an $n$-box that encloses the ambiguity search space, but now one which has its sides parallel to the grid axes. It will be clear that a full decorrelation is achieved when these two $n$-boxes coincide. The results for the seven cases are shown in Table 1. These results clearly show that the precision, the elongation and the decorrelation have improved considerably in all cases. The seven cases have been divided into three groups. This grouping has been done according to the number of very small conditional variances contained in the original spectrum. The first group consists of one case which fails to have small conditional variances in the original spectrum. The second group consists of three cases, all of which have three small conditional variances in the original spectrum. The third group again consists of three cases, but now all have nine small conditional variances in their original spectrum.

The precision of the DD ambiguities of the first case is the poorest of all cases. This is due to the absence of redundancy. The precision of the transformed ambiguities is considerably better. When compared to the other cases, however, it is still at a poor level. This is due to the absence of the small conditional variances in the DD spectrum. For the elongation, we also note a considerable improvement in all cases. Since the elongation has been made smaller, the transformed ambiguity search space is more 'sphere-like' than the original ambiguity search space. Note that the elongations of all transformed search spaces are of about the same order. This is not true however for the original search spaces. The elongation of the original search space of the first case for instance is much smaller than the elongations of the other six cases. This can also be explained by the absence of redundancy. Due to this absence, no functions of the least-squares DD ambiguities can be found which have an extremely high precision. Hence, also the smallest eigenvalue of the ambiguity variance-covariance matrix, the denominator in $e_{\hat{a}}^{2}=\lambda_{\max } / \lambda_{\min }$, will not be so small as to push $e_{\hat{a}}$ to very high values.

In the second group, all three cases have three very small conditional variances in their original spectrum. Note the dramatic improvement in precision of the transformed ambiguities. In all three cases, the precision is of the order of a few cycles only. The precision of the original ambiguities, however, differs greatly between the three cases. When we compare the second with the first case in this group, we note that satellite redundancy has a more favourable effect on the precision of the DD ambiguities than the redundancy coming from the use of a second frequency. Similarly, when we compare the third case with the second, we observe that the use of phase with a longer wavelength improves upon the precision of the original ambiguities. The shape of all three original search spaces is very far from being 'sphere-like'. This is due to the fact that redundancy allows one to find functions of the original ambiguities that have a very high precision.

In the third group, all three cases have nine very small conditional variances in their original spectrum. Again note the improvement in precision of the transformed ambiguities. In all three cases, the precision is now of the order of a few tenths of a cycle. When compared to the three cases of the second group, this improved level of precision is due to the additional six very small conditional variances of the original spectrum. Within this third group, the precision and elongation of the original ambiguities of the first case are considerably worse than those of the second and the third case. This is due to the very small change in time of the relative receiver-satellite geometry. This change is larger for the second case and it has, due to inclusion of code data, almost no effect on the third case. In order to understand the link between decorrelation and precision improvement, consider the ratio of $r_{\hat{z}}$ and $r_{\hat{a}}$. Since the determinant of the ambiguity variance-covariance matrix is invariant under the ambiguity transformation $Z^{*}$, this ratio equals the product of the ratios $\sigma_{\hat{a}_{i}} / \sigma_{\hat{z}_{i}}, i=1, \ldots, n$. This link also shows that the precision improvement of the ambiguities implies an improvement in the correlation coefficient between pairs of ambiguities and vice versa. This is shown in Fig. 7 for the first and third case of group three of Table 1. Shown are the histograms of the absolute values of the sixty-six original (white bars) and transformed (black bars)

Table 1. Precision $\left(\sigma_{\hat{a}}, \sigma_{\hat{z}}\right.$ in cycles $)$, elongation $\left(e_{\hat{a}}, e_{\hat{z}}\right)$ and decorrelation $\left(-\log _{10} r_{\hat{a}},-\log _{10} r_{\hat{z}}\right)$ of original and transformed ambiguities

\begin{tabular}{lccccrrrr}
\hline & $\min \sigma_{\hat{a}}$ & $\max \sigma_{\hat{a}}$ & $\min \sigma_{\hat{z}}$ & $\max \sigma_{\hat{z}}$ & $e_{\hat{a}}$ & $e_{\hat{z}}$ & $-\log _{10} r_{\hat{a}}$ & $-\log _{10} r_{\hat{z}}$ \\
\hline$L_{1}, 4 s v, 1 \mathrm{~s}$ & 213.8 & 641.6 & 88.3 & 384.3 & 42 & 4.4 & 1.3 & 0.002 \\
$L_{1} / L_{2}, 4 s v, 1 \mathrm{~s}$ & 117.8 & 453.7 & 0.9 & 3.5 & 58120 & 5.0 & 13.4 & 0.192 \\
$L_{1}, 7 s v, 1 \mathrm{~s}$ & 98.1 & 285.7 & 2.0 & 2.7 & 23300 & 2.4 & 11.5 & 0.225 \\
widelane, $7 s v, 1 \mathrm{~s}$ & 15.3 & 44.6 & 0.9 & 1.2 & 2870 & 3.1 & 8.8 & 0.259 \\
$L_{1} / L_{2}, 7 s v, 1 \mathrm{~s}$ & 54.1 & 202.0 & 0.2 & 0.3 & 26800 & 5.5 & 33.6 & 1.000 \\
$L_{1} / L_{2}, 7 s v, 60 \mathrm{~s}$ & 0.9 & 3.3 & 0.1 & 0.1 & 445 & 5.1 & 17.6 & 0.928 \\
$L_{1} / L_{2}, 7 s v, 1 \mathrm{~s}$, code & 1.1 & 3.1 & 0.1 & 0.1 & 467 & 6.8 & 17.7 & 0.951 \\
\hline
\end{tabular}




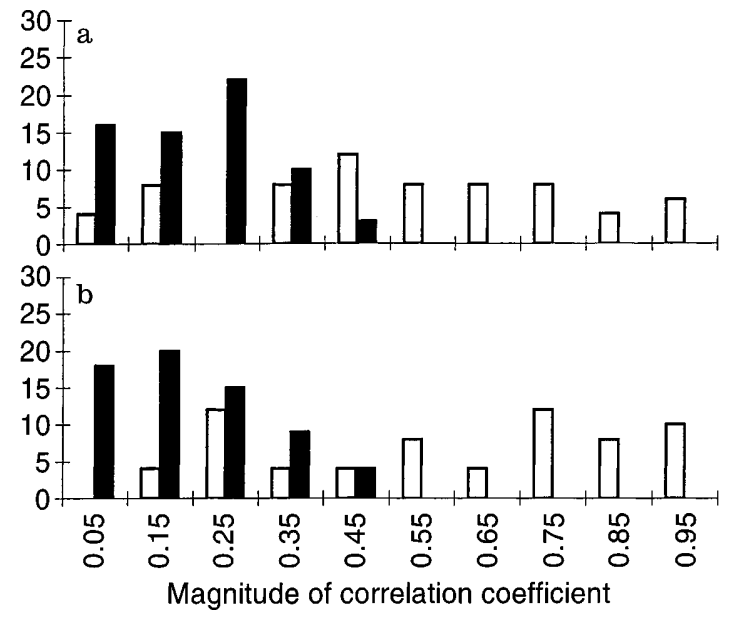

Fig. 7a,b. Histograms of absolute correlation coefficients; a $L_{1} / L_{2}$ phase, $\Delta t=1 \mathrm{~s} ; \mathbf{b} L_{1} / L_{2}$ phase $+\operatorname{code}, \Delta t=1 \mathrm{~s}$

correlation coefficients. It is clear that the ambiguity transformation has indeed succeeded in pushing the largest (in absolute value) correlation coefficients down to much lower values.

\section{Discussion}

In our implementation, the construction of the decorrelating ambiguity transformation and the actual search for the integer least-squares ambiguities are intimately linked through the sequential conditional least-squares adjustment. However, if so desired, one can use different search procedures. Instead of applying it to the original DD ambiguities, it should then be applied to the transformed ambiguities. In fact, since the transformed ambiguities are generally of a very high precision, a simple rounding to the nearest integer of their realvalued least-squares estimates often already gives the correct integer least-squares solution. Here however, a word of caution is in order. Due to correlation that still may exist between the transformed ambiguities, it is not guaranteed that the simple scheme of 'rounding to the nearest integer' will always produce the desired integer least-squares solution.

It is remarked that the decorrelating ambiguity transformation is completely determined by the variance-covariance matrix of the ambiguities. Even the a posteriori variance factor need not be known. This shows that actual measurements are not needed for the decorrelation and that the construction of the ambiguity transformation can commence in principle prior to the actual measurement stage. This is particularly relevant for kinematic positioning and navigation applications. Also, since the relative receiver-satellite geometry will not change too much over small periods of time, the ambiguity transformation computed for one period will be a good approximation to the ambiguity transformation belonging to a neighbouring period. In this way, 'old' ambiguity transformations can be used as 'predictors' for 'new' ambiguity transformations.
In this contribution, we have restricted our performance study to the 'standard' model used for shortbaseline applications. The method, however, is generally applicable. Its use is not restricted to the single- baseline case and can also be applied when additional parameters are estimated. The method is also applicable to cases where the relative receiver-satellite geometry is not used; that is, for cases where code data are used directly together with phase data to determine the integer ambiguities, see Hatch (1982), Euler and Goad (1990) and Euler and Hatch (1994). An analytically based study for this case is given in Teunissen (1996). This study also gives the appropriate decorrelating ambiguity transformations for varying levels of precision of the code and phase measurements.

At present, it is common practice to use longer observation time spans for long baselines. With longer observation time spans, the gain in baseline precision experienced by resolving phase ambiguities gets smaller but is still significant. Furthermore, other parameters of interest, see e.g. Mervart (1995), may be estimated more precisely by the ambiguity resolution. Hence, if these parameters are of interest, integer ambiguity estimation remains a valuable option, so as to determine these parameters with the best possible precision.

\section{Conclusion}

In this contribution, the performance of the least-squares ambiguity decorrelation adjustment method has been presented and discussed. The cause of the poor performance for the search of the DD ambiguities has been explained. We have shown that the method is capable of dramatically improving the integer cycle ambiguity estimation. (Information on the LAMBDA method can be found also on Internet: http://www.geo.tudelft.nl/mgp/lambda/. The computer code and a detailed description of the implementation are available.)

\section{References}

Apostol TM (1969) Calculus, Vol. 2. Wiley, New York Blewitt G (1989) Carrier phase ambiguity resolution for the Global Positioning System applied to geodetic baselines up to $2000 \mathrm{~km}$. J Geophys Res 94. 12 187-12 203

Cocard C, Geiger A (1992) Systematic search for all possible widelanes. Proc 6th Int Geod Symp Satellite Positioning. Columbus, Ohio, 17-20 March 1992.

Dong D-N, Bock Y (1989) Global Positioning System network analysis with phase ambiguity resolution applied to crustal deformation studies in California. J Geophys Res 94: 3949-3966

Euler H-J, Goad C (1990) On optimal filtering of GPS dual frequency observations without using orbit information. Bull Géod 65: 130-143

Euler H-J, Hatch R (1994) Comparison of several AROF kinematic techniques. Proc ION GPS-94 pp 363-370

Euler H-J, Landau H (1992) Fast GPS ambiguity resolution on-thefly for real-time applications. Proc 6th Int Geod Symp Satellite Positioning. Columbus, Ohio, March 17-20, 1992. pp 650-659

Frei E (1991) Rapid differential positioning with the Global Positioning System. Schweizerischen Geodätischen Kommission, Band 44, Bern, Switzerland 
Frei E, Beutler G (1990) Rapid static positioning based on the fast ambiguity resolution approach FARA: theory and first results. Manuscr Geod 15: 325-356

Goad C (1985) Precise relative position determination using the global positioning system carrier phase measurements in a nondifference mode. Proc 1st Int Symp Precise Positioning with Global Positioning System. April 15-19, 1985, Rockville, Maryland. Vol. 1, pp 347-356

Goad C (1992) Robust techniques for determining GPS phase ambiguities. Proc 6th Int Geod Symp Satellite Positioning. Columbus, Ohio, 17-20 March 1992, pp 245-254

Hatch R (1982) The synergism of GPS code and carrier measurements. Proc 3rd Int Geod Symp Satellite Positioning. Las Cruces, New Mexico, 8-12 February, 1982, Vol. 2, pp 12131231

Hatch R (1991) Instantaneous ambiguity resolution. Kinematic systems in geodesy, surveying and remote sensing, IAG Symposia 127 Springer, Berlin Heidelberg New York, pp 299-308

Melbourne WG (1985) The case for ranging in GPS-based geodetic systems. Proc 1st Int Symp Precise Positioning with Global Positioning System. April 15-19, 1985, Rockville, Maryland. Vol. 1. pp 373-386

Mervart L (1995) Ambiguity resolution techniques in geodetic and geodynamic applications of the Global Positioning System. $\mathrm{PhD}$ dissertation, Bern University, Switzerland
Teunissen PJG (1993) Least-squares estimation of the integer GPS ambiguities. LGR series no 6. Delft Geodetic Computing Centre

Teunissen PJG (1995a) The least-squares ambiguity decorrelation adjustment: a method for fast GPS integer ambiguity estimation. J Geod 70: 65-82

Teunissen PJG (1995b) The invertible GPS ambiguity transformations. Manuscr Geod 20: 489-497

Teunissen PJG (1996) An analytical study of ambiguity decorrelation using dual frequency code and carrier phase. J Geod 70: $515-528$

Teunissen PJG, de Jonge PJ, Tiberius CCJM (1994) On the spectrum of the GPS DD ambiguities. In: Proc ION GPS-94. pp 115-224

Teunissen PJG, de Jonge PJ, Tiberius CCJM (1995) A new way to fix carrier-phase ambiguities. GPS-World, April 1995: 58-61

Teunissen PJG, de Jonge PJ, Tiberius CCJM (1996) The volume of the GPS ambiguity search space and its relevance for integer ambiguity resolution. In: Proc ION GPS-96. pp 889-898

Tiberius CCJM, de Jonge PJ (1995) Fast positioning using the LAMBDA-method. In: Proc DSNS-95, Bergen, Norway. 24-28 April 1995. paper no. 30

Wübbena G (1991) Zur Modellerung von GPS Beobachtungen für die hochgenaue Positionsbestimmung. Univ Hannover, Germany 